\title{
Research on Crystal Structure and Fungicidal Activity of the Amide Derivatives Based on the Natural Products Sinapic Acid and Mycophenolic Acid
}

\author{
Zhanfang Chen $\mathbb{D},{ }^{1}$ Hongbin Fang $\mathbb{D},{ }^{1}$ Xuewen Hua $\mathbb{D}^{1},{ }^{1}$ Wenrui Liu $\mathbb{D}^{1},{ }^{1}$ Yi Liu $\mathbb{D}$, ${ }^{1}$ \\ Chenmeng Xue $\left(\mathbb{D},{ }^{1}\right.$ Bingxiang Wang $\left(\mathbb{D},{ }^{2}\right.$ Dzmitry Bazhanau $\left(\mathbb{D},{ }^{1}\right.$ Xiaohe Zhu $\left(\mathbb{D},{ }^{1}\right.$ \\ Man Yuan $\left(D,{ }^{1}\right.$ Jing Ru $\mathbb{D D}^{3}$ and Pengfei Chu $\mathbb{D}^{1}$ \\ ${ }^{1}$ College of Agriculture, Liaocheng University, Liaocheng 252000, China \\ ${ }^{2}$ College of Pharmacy, Liaocheng University, Liaocheng 252000, China \\ ${ }^{3}$ College of Chemistry and Chemical Engineering, Liaocheng University, Liaocheng 252000, China
}

\begin{abstract}
Correspondence should be addressed to Xuewen Hua; huaxuewen906@163.com, Bingxiang Wang; wangbingxiang@lcu.edu.cn, and Pengfei Chu; chupengfei@lcu.edu.cn
\end{abstract}

Received 9 November 2021; Accepted 30 November 2021; Published 21 December 2021

Academic Editor: Wenneng Wu

Copyright (c) 2021 Zhanfang Chen et al. This is an open access article distributed under the Creative Commons Attribution License, which permits unrestricted use, distribution, and reproduction in any medium, provided the original work is properly cited.

\begin{abstract}
Structural optimization based on natural products is an important and effective way to discover new green pesticides. Here, two series of amide derivatives based on sinapic acid and mycophenolic acid were designed in combination with the fungicidal natural product piperlongumine and synthesized by preparing the carboxylic acid into acyl chloride and then reacting with the corresponding aromatic amines, respectively. The resulting structures were successively characterized by ${ }^{1} \mathrm{H} N M R,{ }^{13} \mathrm{C} \mathrm{NMR}$, and HRMS. The crystal structures of molecules I-4 and II-5 were analyzed for structure validation. The in vitro inhibitory activity indicated that most of the target products exhibited fungicidal activity equivalent to or even better than fluopyram against Physalospora piricola. The in vivo fungicidal activity demonstrated that the compounds I-5 and II-4 displayed almost the same preventative activity as carbendazim and fluopyram at $200 \mu \mathrm{g} \mathrm{mL}^{-1}$. The TEM observation revealed that the fungicidal activity of the target molecules against Physalospora piricola may be due to the influence on the mitochondria in the cell structure. These results will provide valuable theoretical guidance for developing the new green fungicides.
\end{abstract}

\section{Introduction}

Agrochemicals are important production materials for agricultural production, and their development plays an important role in ensuring national food security, agricultural product quality, ecological environment safety, and public health [1]. However, the continuous application of traditional chemical fungicides has produced many negative effects including ecological environment pollution, pathogen resistance, and poison for beneficial insects and microorganisms $[2,3]$. Therefore, the development of efficient, safe, low residual, and environmentally friendly green fungicides has become the inevitable trend for pesticide innovation $[4,5]$.

The discovery of lead compounds and the exploration of mechanism of action are the key to the development and innovation of fungicides. Structural optimization based on natural products has become an effective way to develop new green fungicides, which has important guiding significance for practicing new development concepts and promoting green development of agrochemicals [6-11]. For example, coumoxystrobin was successfully developed based on natural products strobilurin and coumarin (Figure 1) [12]. Moreover, natural product strobilurin A-derived 


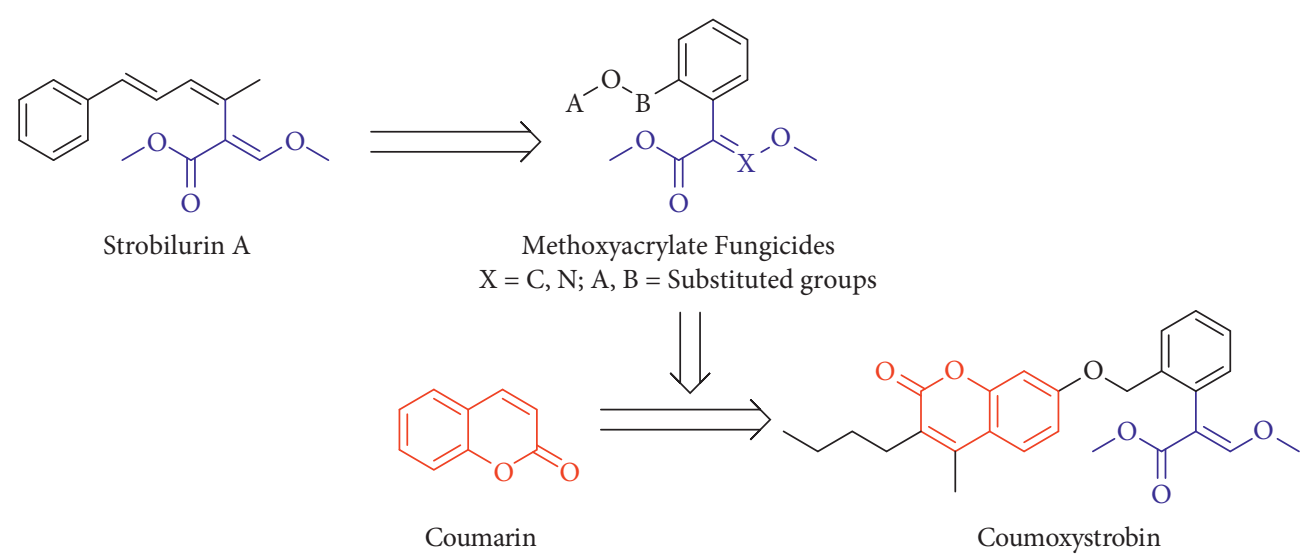

FIGURE 1: Methoxyacrylate fungicides and coumoxystrobin successfully developed based on natural product strobilurin A.

methoxyacrylate fungicides have occupied the top position in the market sales of fungicides [6].

Mycophenolic acid (MPA) was discovered by Gosio in 1893 in a strain of Penicillium fungus and was found to possess broad biological activity such as antifungal, antiviral, anticancer, and antipsoriasis properties $[13,14]$. Sinapic acid is widely distributed in the plants and belongs to hydroxycinnamic acid. Furthermore, commercial fungicides dimethomorph, pyrimorph, and flumorph were successively developed based on natural product cinnamic acid (Figure 2) [15-18]. In this project, combined with the structure of fungicidal natural product piperlongumine $[19,20]$, two series of amide compounds based on natural products sinapic acid and mycophenolic acid were designed, synthesized, and evaluated their fungicidal activity against the common agricultural pathogens (Figure 3).

\section{Experimental Materials and Methods}

2.1. Materials and Equipment. The materials and reagents used in the organic synthesis reactions were of analytical grade and purchased from Energy Chemical and Bide Pharmatech Ltd. Melting points were measured on a X-5 binocular microscope (Yuhua Co., Ltd., China). ${ }^{1} \mathrm{H}$ NMR and ${ }^{13} \mathrm{C}$ NMR were provided on a AVANCE NEO-500 MHz spectrometer (Bruker, Germany). HRMS was recorded on a Xevo G2-XS QTof spectrometer (Waters, USA). X-ray crystal structure was determined on a D8 Venture diffractometer (Bruker, Germany). The purification of target compounds was performed by the column chromatography on silica gel (200-300 mesh).

\subsection{Preparation of the Target Molecules.} (E)-2,6-dimethoxy-4-(3-oxo-3-(phenylamino)prop-1-en-1yl)phenyl acetate (I-1) was synthesized as follows. Thionyl chloride $(2.38 \mathrm{~g}, 20.0 \mathrm{mmol})$ was added dropwise to a mixture of (E)-3-(4-acetoxy-3,5-dimethoxyphenyl) acrylic acid (1.06 g, $4.0 \mathrm{mmol}$ ) and DMF (3drops) in DCM (20 mL). The reaction was stirred for $6 \mathrm{~h}$ at room temperature and concentrated in vacuo to sufficiently remove the solvent and excess thionyl chloride. The resulting acyl chloride was immediately dissolved in anhydrous DCM $(3 \mathrm{~mL})$ and then added dropwise at room temperature to a mixture of aniline $(0.45 \mathrm{~g}, 4.8 \mathrm{mmol})$ and trimethylamine $(0.81 \mathrm{~g}, 8.0 \mathrm{mmol})$ in anhydrous DCM (25 mL). The reaction was stirred overnight and poured into a mixture of DCM and water $(150 \mathrm{~mL}, \mathrm{v} /$ $\mathrm{v}=1 / 1)$. The DCM layer was separated by extraction and washed successively with dilute hydrochloric acid and potassium carbonate aqueous solution. The obtained solution was dried over anhydrous sodium sulfate, filtered, and concentrated. The provided residue was purified by silica gel chromatography using petroleum ether/ethyl acetate ( $\mathrm{v} /$ $\mathrm{V}=1 / 1$ ) as eluent to provide I-1 (yield 68\%). Molecules I2-I-5 and II-1-II-5 were prepared similarly.

(E)-2,6-dimethoxy-4-(3-oxo-3-(phenylamino)prop-1en-1-yl)phenyl acetate (I-1): white solid, yield 68\%, m.p. 125-127 ${ }^{\circ} \mathrm{C} .{ }^{1} \mathrm{H}$ NMR $\left(500 \mathrm{MHz}, \mathrm{CDCl}_{3}\right) \delta 7.67$ (s, $\left.1 \mathrm{H}, \mathrm{NH}\right)$, 7.63-7.57 (m, 3H, Ph-H, and CH), $7.34(\mathrm{t}, J=7.9 \mathrm{~Hz}, 2 \mathrm{H}, \mathrm{Ph}-$ $\mathrm{H}), 7.12$ (t, J=7.4 Hz, 1H, Ph-H), 6.68 (s, 2H, Ph-H), 6.39 (d, $J=15.4 \mathrm{~Hz}, 1 \mathrm{H}, \mathrm{CH}), 3.78\left(\mathrm{~s}, 6 \mathrm{H},\left(\mathrm{OCH}_{3}\right)_{2}\right), 2.37$ (s, 3H, $\left.\mathrm{COCH}_{3}\right) .{ }^{13} \mathrm{C} \mathrm{NMR}\left(126 \mathrm{MHz}, \mathrm{CDCl}_{3}\right) \delta 169.0,163.8,152.3$, $141.7,138.2$, 133.2, 130.1, 129.1, 124.4, 121.4, 119.8, 104.7, 56.2, 20.5. Found, $m / z: 342.1333[\mathrm{M}+\mathrm{H}]^{+} \cdot \mathrm{C}_{19} \mathrm{H}_{20} \mathrm{NO}_{5}$. Calculated, $m / z: 342.1336$.

(E)-2,6-dimethoxy-4-(3-oxo-3-((2-(trifluoromethyl) phenyl)amino)prop-1-en-1-yl)phenyl acetate (I-2): white solid, yield $60 \%$, m.p. $143-145^{\circ} \mathrm{C}$. ${ }^{1} \mathrm{H}$ NMR $(500 \mathrm{MHz}$, $\left.\mathrm{CDCl}_{3}\right) d 8.37(\mathrm{~d}, J=7.9 \mathrm{~Hz}, 1 \mathrm{H}, \mathrm{Ph}-\mathrm{H}), 7.68(\mathrm{~d}, J=15.4 \mathrm{~Hz}$, $1 \mathrm{H}, \mathrm{CH}), 7.66-7.57(\mathrm{~m}, 3 \mathrm{H}, \mathrm{NH}$, and $\mathrm{Ph}-\mathrm{H}), 7.27-7.24(\mathrm{~m}$, $1 \mathrm{H}, \mathrm{Ph}-\mathrm{H}), 6.80$ (s, 2H, Ph-H), 6.47 (d, J=15.4 Hz, 1H, CH), $3.87\left(\mathrm{~s}, 6 \mathrm{H},\left(\mathrm{OCH}_{3}\right)_{2}\right), 2.36\left(\mathrm{~s}, 3 \mathrm{H}, \mathrm{COCH}_{3}\right) .{ }^{13} \mathrm{C} \mathrm{NMR}$ $\left(126 \mathrm{MHz} \mathrm{CDCl}_{3}\right) \delta 168.6,163.8,152.5,143.2,135.4,135.4$, $133.0,132.6,130.4,126.1\left(\mathrm{q}, J_{C-F}=5.4 \mathrm{~Hz}\right), 124.5,124.4$, $124.2\left(\mathrm{q}, J_{C-F}=273.4 \mathrm{~Hz}\right), 120.5,104.7,56.3,20.5$. Found, $m /$ $z: 410.1208 \quad[\mathrm{M}+\mathrm{H}]^{+} . \quad \mathrm{C}_{20} \mathrm{H}_{19} \mathrm{~F}_{3} \mathrm{NO}_{5}$. Calculated, $m / z$ : 410.1210 .

(E)-4-(3-((2,3-dichlorophenyl)amino)-3-oxoprop-1-en1-yl)-2,6-dimethoxyphenyl acetate (I-3) : white solid, yield

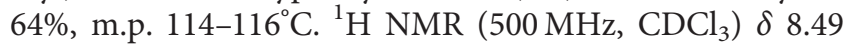
(dd, $J=7.5,2.2 \mathrm{~Hz}, 1 \mathrm{H}, \mathrm{Ph}-\mathrm{H}), 7.89$ (s, 1H, NH), 7.67 (d, $J=15.4 \mathrm{~Hz}, 1 \mathrm{H}, \mathrm{CH}), 7.27-7.23(\mathrm{~m}, 2 \mathrm{H}, \mathrm{Ph}-\mathrm{H}), 6.78(\mathrm{~s}, 2 \mathrm{H}$, $\mathrm{Ph}-\mathrm{H}), 6.53$ (d, J=15.4 Hz, 1H, CH), $3.86\left(\mathrm{~s}, 6 \mathrm{H},\left(\mathrm{OCH}_{3}\right)_{2}\right)$, $2.36\left(\mathrm{~s}, 3 \mathrm{H}, \mathrm{COCH}_{3}\right) .{ }^{13} \mathrm{C} \mathrm{NMR}\left(126 \mathrm{MHz}, \mathrm{CDCl}_{3}\right) \delta 168.6$, 


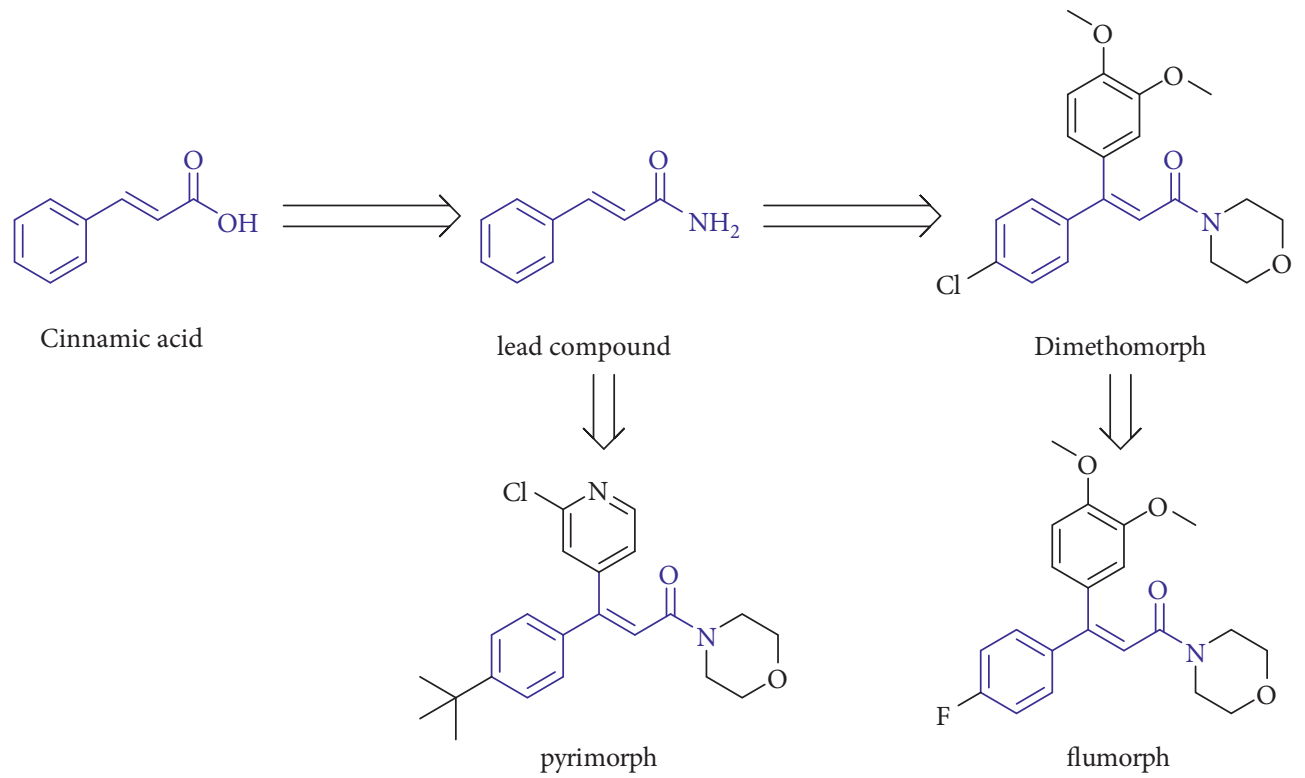

Figure 2: Cinnamic acid amide fungicides successfully developed based on natural product cinnamic acid.<smiles>COc1cc(/C=C/C(=O)O)cc(OC)c1O</smiles>

Sinapic acid<smiles>COc1c(C)c2c(c(O)c1C/C=C(\C)CCC(=O)O)C(=O)OC2</smiles>

Mycophenolic acid<smiles>COc1cc(/C=C/C(=O)N2CCC=CC2=O)cc(OC)c1OC</smiles>

\section{(Fungicidal natural product)}

Structural modification<smiles>COc1cc(/C=C/C(=O)N[Al])cc(OC)c1OC(C)=O</smiles>

Target compounds I<smiles>COc1c(C)c2c(c(OC(C)=O)c1C/C=C(\C)CCC(=O)N[Al])C(=O)OC2</smiles>

Target compounds II

Figure 3: Molecular design strategy in this project.

$163.7,152.4,143.2,136.4,132.8,132.6,130.4,127.9,125.3$, $121.2,120.6,119.6,104.7,56.2,20.5$. Found, $m / z: 410.0555$ $[\mathrm{M}+\mathrm{H}]^{+} \cdot \mathrm{C}_{19} \mathrm{H}_{18} \mathrm{Cl}_{2} \mathrm{NO}_{5}$. Calculated, $m / z: 410.0557$.

(E)-2,6-dimethoxy-4-(3-((2-methoxy-5-methylphenyl) amino)-3-oxoprop-1-en-1-yl)phenyl acetate (I-4): white solid, yield $72 \%$, m.p. $153-155^{\circ} \mathrm{C}$. ${ }^{1} \mathrm{H}$ NMR $(500 \mathrm{MHz}$, $\left.\mathrm{CDCl}_{3}\right) \delta 8.35(\mathrm{~s}, 1 \mathrm{H}, \mathrm{NH}), 7.93(\mathrm{~s}, 1 \mathrm{H}, \mathrm{Ph}-\mathrm{H}), 7.65$ (d, $J=15.4 \mathrm{~Hz}, 1 \mathrm{H}, \mathrm{CH}), 6.86(\mathrm{dd}, J=8.2,1.4 \mathrm{~Hz}, 1 \mathrm{H}, \mathrm{Ph}-\mathrm{H})$, 6.80-6.78 (m, 3H, Ph-H), 6.52 (d, J=15.4 Hz, 1H, CH), 3.89 $\left(\mathrm{s}, 3 \mathrm{H}, \mathrm{OCH}_{3}\right), 3.87\left(\mathrm{~s}, 6 \mathrm{H},\left(\mathrm{OCH}_{3}\right)_{2}\right), 2.35$ (s, 3H, $\left.\mathrm{COCH}_{3}\right)$, $2.33\left(\mathrm{~s}, 3 \mathrm{H}, \mathrm{Ph}-\mathrm{CH}_{3}\right) .{ }^{13} \mathrm{C} \mathrm{NMR}\left(126 \mathrm{MHz}, \mathrm{CDCl}_{3}\right) \delta 168.6$, $163.4,152.4,145.9,141.6,133.2,130.8,130.1,127.5,124.1$, $121.7,120.7,109.8,104.6,56.2,55.9,21.0,20.5$. Found, $m / z$ : $386.1594[\mathrm{M}+\mathrm{H}]^{+} . \mathrm{C}_{21} \mathrm{H}_{24} \mathrm{NO}_{6}$. Calculated, $m / z: 386.1598$.

(E)-4-(3-((3-isopropoxyphenyl)amino)-3-oxoprop-1en-1-yl)-2,6-dimethoxyphenyl acetate (I-5): white solid, yield $68 \%$, m.p. $122-124^{\circ} \mathrm{C} .{ }^{1} \mathrm{H}$ NMR $\left(500 \mathrm{MHz}, \mathrm{CDCl}_{3}\right) \delta$ 7.61 (d, J=15.4 Hz, 1H, CH), 7.51 (s, 1H, Ph-H), 7.41 (s, 1H, $\mathrm{NH}), 7.21(\mathrm{t}, J=8.1 \mathrm{~Hz}, 1 \mathrm{H}, \mathrm{Ph}-\mathrm{H}), 7.03(\mathrm{~d}, J=7.8 \mathrm{~Hz}, 1 \mathrm{H}$, $\mathrm{Ph}-\mathrm{H}), 6.71$ (s, 2H, Ph-H), 6.67 (dd, $J=8.2,1.8 \mathrm{~Hz}, 1 \mathrm{H}, \mathrm{Ph}-$ $\mathrm{H}), 6.40$ (d, $J=15.4 \mathrm{~Hz}, 1 \mathrm{H}, \mathrm{CH}), 4.57$ (hept, $J=6.1 \mathrm{~Hz}, 1 \mathrm{H}$, $\left.\mathrm{CH}\left(\mathrm{CH}_{3}\right)_{2}\right), 3.81\left(\mathrm{~s}, 6 \mathrm{H},\left(\mathrm{OCH}_{3}\right)_{2}\right), 2.36$ (s, 3H, $\left.\mathrm{COCH}_{3}\right)$,

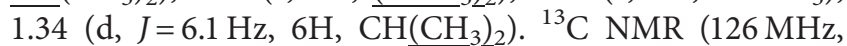
$\left.\mathrm{CDCl}_{3}\right) \delta 168.9,163.7,158.6,152.3,149.3,141.9,139.3,133.1$, 130.1, 129.7, 121.3, 112.3, 111.8, 104.6, 70.0, 56.2, 22.1, 20.5. Found, $m / z: 400.1753[\mathrm{M}+\mathrm{H}]^{+} . \mathrm{C}_{22} \mathrm{H}_{26} \mathrm{NO}_{6}$. Calculated, $m / z$ : 400.1755 .

(E)-6-methoxy-7-methyl-5-(3-methyl-6-oxo-6-(phenylamino)hex-2-en-1-yl)-3-oxo-1,3-dihydroisobenzofuran-4yl acetate (II-1) : white solid, yield $66 \%$, m.p. $160-162^{\circ} \mathrm{C} .{ }^{1} \mathrm{H}$ NMR $\left(500 \mathrm{MHz}, \mathrm{CDCl}_{3}\right) \delta 7.53(\mathrm{~s}, 1 \mathrm{H}, \mathrm{NH}), 7.33$ (d, $J=7.8 \mathrm{~Hz}, 2 \mathrm{H}, \mathrm{Ph}-\mathrm{H}), 7.18(\mathrm{t}, J=7.9 \mathrm{~Hz}, 2 \mathrm{H}, \mathrm{Ph}-\mathrm{H}), 7.01(\mathrm{t}$, 
$J=7.4 \mathrm{~Hz}, 1 \mathrm{H}, \mathrm{Ph}-\mathrm{H}), 5.15(\mathrm{t}, J=6.9 \mathrm{~Hz}, 1 \mathrm{H}, \mathrm{CH}), 5.01(\mathrm{~s}$, $\left.2 \mathrm{H}, \mathrm{OCH}_{2} \mathrm{Ph}\right), 3.70\left(\mathrm{~s}, 3 \mathrm{H}, \mathrm{OCH}_{3}\right), 3.35(\mathrm{~d}, J=7.1 \mathrm{~Hz}, 2 \mathrm{H}$, $\left.\mathrm{PhCH}_{2}\right), 2.40\left(\mathrm{~s}, 3 \mathrm{H}, \mathrm{COCH}_{3}\right), 2.38-2.35\left(\mathrm{~m}, 4 \mathrm{H}, \mathrm{CH}_{2} \mathrm{CH}_{2}\right)$, $2.06\left(\mathrm{~s}, 3 \mathrm{H}, \mathrm{Ph}-\mathrm{CH}_{3}\right), 1.81\left(\mathrm{~s}, 3 \mathrm{H}, \mathrm{CH}_{3}\right) .{ }^{13} \mathrm{C}$ NMR $(126 \mathrm{MHz}$, $\left.\mathrm{CDCl}_{3}\right) \delta 170.9,169.9,168.4,162.6,146.1,145.5,138.1,134.6$, $129.2,128.5,123.7,123.0,122.5,119.6,113.2,68.4,61.2,36.1$, 34.4, 23.5, 20.8, 16.8, 11.7. Found, $m / z: 438.1907[\mathrm{M}+\mathrm{H}]^{+}$. $\mathrm{C}_{25} \mathrm{H}_{28} \mathrm{NO}_{6}$. Calculated, $m / z: 438.1911$.

(E)-6-methoxy-7-methyl-5-(3-methyl-6-oxo-6-((2-(trifluoromethyl)phenyl) amino)hex-2-en-1-yl)-3-oxo-1,3dihydroisobenzofuran-4-yl acetate (II-2): white solid, yield $70 \%$, m.p. $93-95^{\circ} \mathrm{C} .{ }^{1} \mathrm{H}$ NMR $\left(500 \mathrm{MHz}, \mathrm{CDCl}_{3}\right) \delta 8.09(\mathrm{~d}$, $J=7.6 \mathrm{~Hz}, 1 \mathrm{H}, \mathrm{Ph}-\mathrm{H}), 7.58(\mathrm{~d}, J=7.8 \mathrm{~Hz}, 1 \mathrm{H}, \mathrm{Ph}-\mathrm{H}), 7.51(\mathrm{t}$, $J=7.8 \mathrm{~Hz}, 1 \mathrm{H}, \mathrm{Ph}-\mathrm{H}), 7.38(\mathrm{~s}, 1 \mathrm{H}, \mathrm{NH}), 7.21(\mathrm{t}, J=7.6 \mathrm{~Hz}$, $1 \mathrm{H}, \mathrm{Ph}-\mathrm{H}), 5.19(\mathrm{t}, J=6.4 \mathrm{~Hz}, 1 \mathrm{H}, \mathrm{CH}), 5.11(\mathrm{~s}, 2 \mathrm{H}$, $\left.\mathrm{OCH}_{2} \mathrm{Ph}\right), 3.77\left(\mathrm{~s}, 3 \mathrm{H}, \mathrm{OCH}_{3}\right), 3.36(\mathrm{~d}, J=6.8 \mathrm{~Hz}, 2 \mathrm{H}$, $\left.\mathrm{PhCH}_{2}\right), 2.51-2.44\left(\mathrm{~m}, 2 \mathrm{H}, \mathrm{CH}_{2}\right), 2.41(\mathrm{t}, J=7.4 \mathrm{~Hz}, 2 \mathrm{H}$, $\mathrm{CH}_{2}$ ), 2.37 (s, 3H, $\left.\mathrm{COCH}_{3}\right), 2.19$ (s, 3H, Ph- $\left.\mathrm{CH}_{3}\right), 1.83$ (s, $\left.3 \mathrm{H}, \mathrm{CH}_{3}\right) .{ }^{13} \mathrm{C}$ NMR $\left(126 \mathrm{MHz}, \mathrm{CDCl}_{3}\right) \delta 170.9,169.0,168.2$, $162.6,146.2,145.9,135.2,134.3,132.8,129.1,126.0\left(\mathrm{q}, J_{C-}\right.$ $\left.{ }_{F}=5.4 \mathrm{~Hz}\right), 125.1,124.8,124.5,122.9,122.8,113.5,68.3,61.2$, 36.1, 34.7, 23.6, 20.5, 16.3, 11.8. Found, $m / z: 506.1781$ $[\mathrm{M}+\mathrm{H}]^{+} . \mathrm{C}_{26} \mathrm{H}_{27} \mathrm{~F}_{3} \mathrm{NO}_{6}$. Calculated, $m / z: 506.1785$.

(E)-5-(6-((2,3-dichlorophenyl)amino)-3-methyl-6-oxohex-2-en-1-yl)-6-methoxy-7-methyl-3-oxo-1,3-dihy-

droisobenzofuran-4-yl acetate (II-3): white solid, yield 67\%,

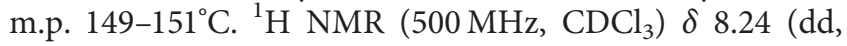
$J=6.5,2.9 \mathrm{~Hz}, 1 \mathrm{H}, \mathrm{Ph}-\mathrm{H}), 7.67$ (s, 1H, NH), 7.18 (s, $1 \mathrm{H}, \mathrm{Ph}-$ $\mathrm{H}), 7.17$ (dd, $1 \mathrm{H}, J=8.5,3.5 \mathrm{~Hz}, \mathrm{Ph}-\mathrm{H}), 5.20(\mathrm{td}, J=6.9$, $1.1 \mathrm{~Hz}, 1 \mathrm{H}, \mathrm{CH}), 5.12\left(\mathrm{~s}, 2 \mathrm{H}, \mathrm{OCH}_{2} \mathrm{Ph}\right), 3.74\left(\mathrm{~s}, 3 \mathrm{H}, \mathrm{OCH}_{3}\right)$, $3.36\left(\mathrm{~d}, J=6.8 \mathrm{~Hz}, 2 \mathrm{H}, \mathrm{PhCH}_{2}\right), 2.51\left(\mathrm{t}, J=7.6 \mathrm{~Hz}, 2 \mathrm{H}, \mathrm{CH}_{2}\right)$, $2.42\left(\mathrm{t}, J=7.4 \mathrm{~Hz}, 2 \mathrm{H}, \mathrm{CH}_{2}\right), 2.39$ (s, 3H, $\left.\mathrm{COCH}_{3}\right), 2.16$ (s, $\left.3 \mathrm{H}, \mathrm{Ph}-\mathrm{CH}_{3}\right), 1.84\left(\mathrm{~s}, 3 \mathrm{H}, \mathrm{CH}_{3}\right),{ }^{13} \mathrm{C}$ NMR $(126 \mathrm{MHz}$, $\left.\mathrm{CDCl}_{3}\right) \delta 170.8,169.1,168.2,162.6,146.2,145.9,136.2$, $134.2,132.6,129.0,127.7,125.1,122.9,119.7,113.5,68.3$, $61.1,36.2,34.6,23.6,20.6,16.5,11.8$. Found, $m / z: 506.1129$ $[\mathrm{M}+\mathrm{H}]^{+} . \mathrm{C}_{25} \mathrm{H}_{26} \mathrm{Cl}_{2} \mathrm{NO}_{6}$. Calculated, $m / z: 506.1132$.

(E)-6-methoxy-5-(6-((2-methoxy-5-methylphenyl) amino)-3-methyl-6-oxohex-2-en-1-yl)-7-methyl-3-oxo-1,3dihydroisobenzofuran-4-yl acetate (II-4): white solid, yield $74 \%$, m.p. $113-115^{\circ} \mathrm{C} .{ }^{1} \mathrm{H}$ NMR $\left(500 \mathrm{MHz}, \mathrm{CDCl}_{3}\right) \delta 8.14$ (s, $1 \mathrm{H}, \mathrm{NH}), 7.69(\mathrm{~s}, 1 \mathrm{H}, \mathrm{Ph}-\mathrm{H}), 6.79$ (dd, $J=8.2,1.1 \mathrm{~Hz}, 1 \mathrm{H}, \mathrm{Ph}-$ $\mathrm{H}), 6.71(\mathrm{~d}, J=8.3 \mathrm{~Hz}, 1 \mathrm{H}, \mathrm{Ph}-\mathrm{H}), 5.16(\mathrm{t}, J=6.4 \mathrm{~Hz}, 1 \mathrm{H}$, $\mathrm{CH}), 5.10\left(\mathrm{~s}, 2 \mathrm{H}, \mathrm{OCH}_{2} \mathrm{Ph}\right), 3.81\left(\mathrm{~s}, 3 \mathrm{H}, \mathrm{OCH}_{3}\right), 3.76(\mathrm{~s}, 3 \mathrm{H}$, $\left.\mathrm{OCH}_{3}\right), 3.35$ (d, $\left.J=6.8 \mathrm{~Hz}, 2 \mathrm{H}, \mathrm{PhCH}_{2}\right), 2.46-2.38(\mathrm{~m}, 4 \mathrm{H}$, $\left.\mathrm{CH}_{2} \mathrm{CH}_{2}\right), 2.37$ (s, 3H, $\left.\mathrm{COCH}_{3}\right), 2.27\left(\mathrm{~s}, 3 \mathrm{H}, \mathrm{Ph}-\mathrm{CH}_{3}\right), 2.17$ (s, $\left.3 \mathrm{H}, \mathrm{Ph}-\mathrm{CH}_{3}\right), 1.83\left(\mathrm{~s}, 3 \mathrm{H}, \mathrm{CH}_{3}\right) .{ }^{13} \mathrm{C}$ NMR $(126 \mathrm{MHz}$, $\left.\mathrm{CDCl}_{3}\right) \delta 170.5,169.0,168.3,162.6,146.1,145.9,145.7$, $134.7,130.4,129.2,127.3,123.6,122.9,122.3,120.4,113.4$, $109.6,68.3,61.2,55.7,36.4,34.8,23.6,20.9,20.5,16.5,11.8$. Found, $m / z: 506.1129[\mathrm{M}+\mathrm{H}]^{+}$. $\mathrm{C}_{27} \mathrm{H}_{32} \mathrm{NO}_{7}$. Calculated, $m / z$ : 506.1132 .

(E)-5-(6-((3-isopropoxyphenyl)amino)-3-methyl-6oxohex-2-en-1-yl)-6-methoxy-7-methyl-3-oxo-1,3-dihydroisobenzofuran-4-yl acetate (II-5): white solid, yield 58\%,

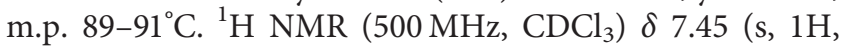
$\mathrm{NH}), 7.12(\mathrm{~s}, 1 \mathrm{H}, \mathrm{Ph}-\mathrm{H}), 7.05$ (t, $J=8.1 \mathrm{~Hz}, 1 \mathrm{H}, \mathrm{Ph}-\mathrm{H}), 6.75$ (dd, $J=8.0,0.9 \mathrm{~Hz}, 1 \mathrm{H}, \mathrm{Ph}-\mathrm{H}), 6.56$ (dd, $J=8.2,1.8 \mathrm{~Hz}, 1 \mathrm{H}$, $\mathrm{Ph}-\mathrm{H}), 5.15(\mathrm{t}, J=6.9 \mathrm{~Hz}, 1 \mathrm{H}, \mathrm{CH}), 5.05\left(\mathrm{~s}, 2 \mathrm{H}, \mathrm{OCH}_{2} \mathrm{Ph}\right)$, $4.48(\mathrm{dt}, J=12.1,6.1 \mathrm{~Hz}, 1 \mathrm{H}, \mathrm{OCH}), 3.71\left(\mathrm{~s}, 3 \mathrm{H}, \mathrm{OCH}_{3}\right), 3.35$ (d, $\left.J=7.1 \mathrm{~Hz}, 2 \mathrm{H}, \mathrm{PhCH}_{2}\right), 2.41\left(\mathrm{~s}, 3 \mathrm{H}, \mathrm{COCH}_{3}\right), 2.40-2.31$ (m, $4 \mathrm{H}, \mathrm{CH}_{2} \mathrm{CH}_{2}$ ), 2.08 (s, 3H, Ph- $\left.\mathrm{CH}_{3}\right), 1.81\left(\mathrm{~s}, 3 \mathrm{H}, \mathrm{CH}_{3}\right.$ ), $1.30\left(\mathrm{~d}, J=6.1 \mathrm{~Hz}, 6 \mathrm{H}, \mathrm{CH}\left(\mathrm{CH}_{3}\right)_{2}\right) .{ }^{13} \mathrm{C}$ NMR $(126 \mathrm{MHz}$, $\left.\mathrm{CDCl}_{3}\right) \delta 170.9,169.8,168.4,162.5,158.2,146.2,145.6$, $139.2,134.6,129.2,129.1,123.0,122.5,113.2,111.8,111.6$, $107.1,70.0,68.4,61.2,36.2,34.4,23.5,22.0,20.8,16.8,11.7$. Found, $m / z: 496.2326[\mathrm{M}+\mathrm{H}]^{+} . \mathrm{C}_{28} \mathrm{H}_{34} \mathrm{NO}_{7}$. Calculated, $m /$ $z: 496.2330$.

2.3. X-Ray Crystal Structure Determination. The crystals of the target compounds I-4 and II-5 were cultivated from a mixed solvent of methanol, ethyl acetate, and n-hexane, respectively. All measurements were made on a Bruker D8 Venture diffractometer with $M o-K \alpha$ radiation $(\lambda=0.71073 \AA)$. The crystal data of the compound I-4 were collected at $298 \mathrm{~K}$, and the colorless crystal is of monoclinic system, space group $C 2 / c$, with $a=28.208$ (3), $b=11.1670$ (12), $c=14.0948$ (14) $\AA, \alpha=90^{\circ}, \beta=114.242(4)^{\circ}, \gamma=90^{\circ}$, $V=4048.3$ (7) $\AA^{3}, Z=8, F(000)=1712$, density (calculated $)=1.324 \mathrm{~g} / \mathrm{cm}^{3}$, and linear absorption coefficient $0.100 \mathrm{~mm}^{-1}$. All of the non-H atoms were refined anisotropically by full-matrix least-squares to give the final $R=0.0493$ and $w R=0.1153 \quad\left(w=1 /\left[\sigma^{2}\left(F_{o}^{2}\right)+(0.0465 P)\right.\right.$ $\left.{ }^{2}+2.2020 P\right]$, where $\left.P=\left(F_{o}{ }^{2}+2 F_{c}{ }^{2}\right) / 3\right)$ with $S=1.064$ using the SHELXL program. The crystal data of the compound II-5 were collected at 298(2) $\mathrm{K}$, and the colorless crystal is of monoclinic system, space group $P 2_{1} / n$, with $a=15.3461$ (16), $b=10.3766$ (11), $c=16.0494$ (17) $\AA, \alpha=90^{\circ}, \beta=95.683$ (2) ${ }^{\circ}$, $\gamma=90^{\circ}, V=2543.2(5) \AA^{3}, Z=4, F(000)=1056$, density (calculated) $=1.294 \mathrm{~g} / \mathrm{cm}^{3}$, and linear absorption coefficient $0.093 \mathrm{~mm}^{-1}$. All of the non- $\mathrm{H}$ atoms were refined anisotropically by full-matrix least-squares to give the final $R=0.0625 \quad$ and $w R=0.1565 \quad\left(w=1 /\left[\sigma^{2}\left(F_{o}{ }^{2}\right)+(0.0001 P)\right.\right.$ $\left.{ }^{2}+0.9874 P\right]$, where $\left.P=\left(F_{o}^{2}+2 F_{c}^{2}\right) / 3\right)$ with $S=1.044$ using the SHELXL program. The crystal structures were solved by direct methods with SHELXS-2014/6 program.

2.4. Fungicidal Activity Measurement. With fluopyram and carbendazim as positive controls, the mycelial growth inhibition method was used to determine the in vitro inhibitory activities of the target compounds against common agricultural pathogens according to the previously reported procedures [21,22], and each treatment was repeated at least three times. The tested pathogens include Rhizoctonia solani (RS), Gibberella zeae (GZ), Botrytis cinerea (BC), Physalospora piricola (PP), Cercospora circumscissa Sacc. (CS), Colletotrichum capsici (CC), Alternaria kikuchiana Tanaka (AK), and Alternaria sp. (AS). The in vivo fungicidal activity of compounds I-5 and II-4 against Physalospora piricola was performed on apples referring to literature methods [23]. The target molecule $(5.0 \mathrm{mg})$ was dissolved in dimethyl sulfoxide $(30 \mu \mathrm{L})$ and diluted with $0.1 \%$ Tween- 80 aqueous solution to provide the test stock solution $\left(200 \mu \mathrm{g} \cdot \mathrm{mL}^{-1}\right)$, which was sprayed with the same volume on healthy apples. Subsequently, the fungi cake containing Physalospora piricola with a diameter of $7 \mathrm{~mm}$ was inoculated. After cultivation at $25^{\circ} \mathrm{C}$ for 5 days, the average lesion area was 


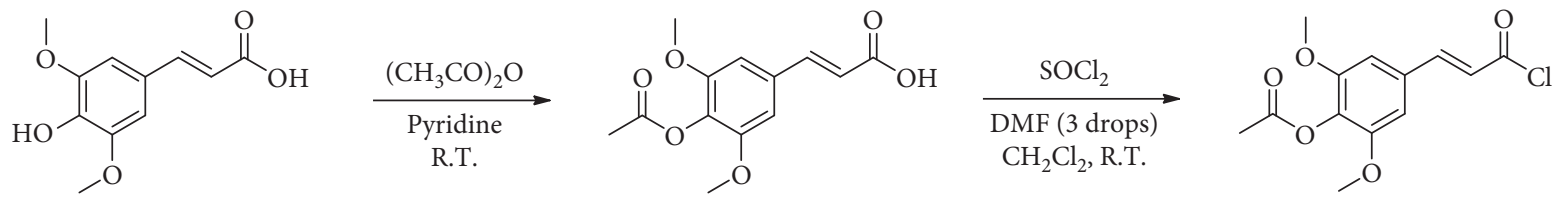
Sinapic acid 1 2

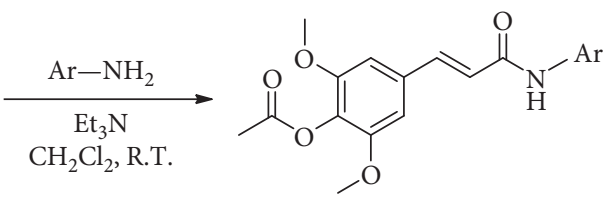

I-1-I-5

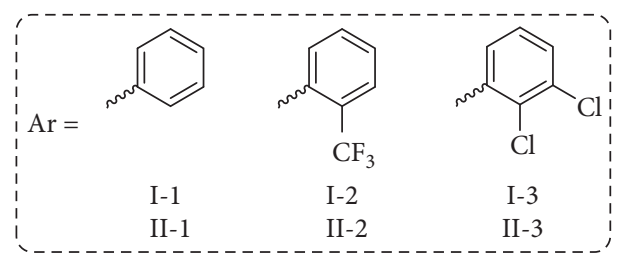

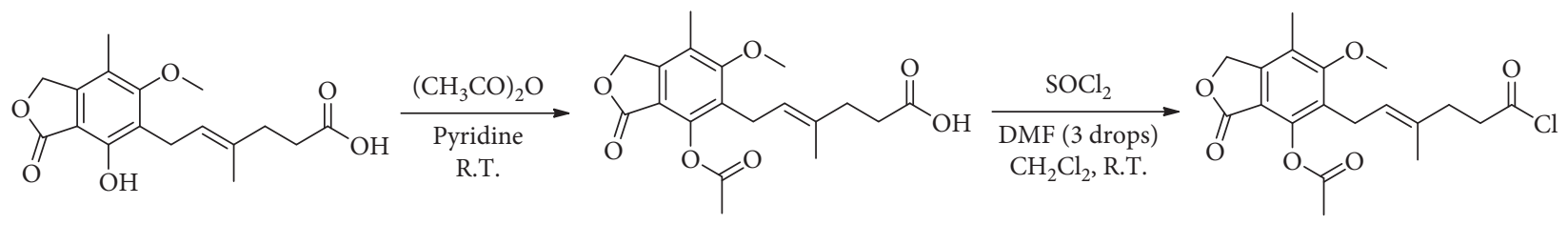
Mycophenolic acid

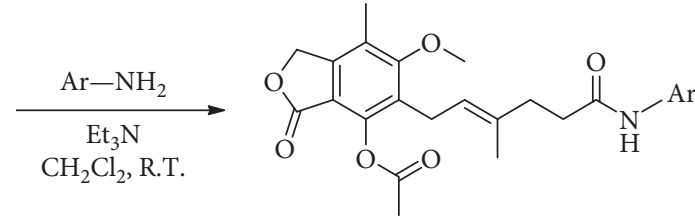

II-1-II-5

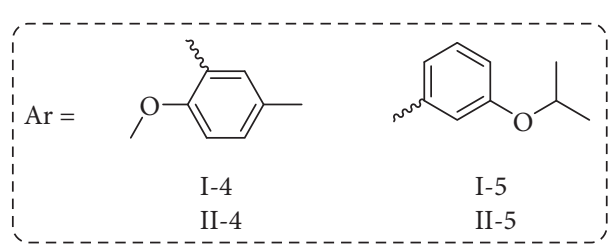

Scheme 1: The synthetic procedures for the target molecules I and II.

measured to calculate the preventative activity. Each in vivo fungicidal activity screening was carried out for at least five repeats.

2.5. Transmission Electron Microscope (TEM) Investigation. Physalospora piricola hyphae were obtained by incubation in $\mathrm{PDB}$ medium at $25^{\circ} \mathrm{C}$ for $72 \mathrm{~h}$ and centrifugation at $7000 \mathrm{rpm}$ for $3 \mathrm{~min}$, which were then resuspended in PDB medium to treat with compounds I-5 and II-4 (200 $\mu \mathrm{g}$ $\cdot \mathrm{mL}^{-1}$ ) for $24 \mathrm{~h}$, respectively. Subsequently, the treated hyphae were provided by centrifugation and fixed with $2.5 \%$ glutaraldehyde. The ultrastructure observation of the hyphae treated with compounds I-5 and II-4 $\left(200 \mu \mathrm{g} \cdot \mathrm{mL}^{-1}\right)$ was performed by Shiyanjia Lab on a TEM according to the standard procedures.

\section{Results and Discussion}

3.1. Organic Synthesis. Herein, the important intermediates and target molecules I-1-I-5 and II-1-II-5 were provided referring to the reported procedures (Scheme 1). In the preparation of target compound I-1-I-5, the phenolic hydroxyl group in sinapic acid was firstly reacted with acetic anhydride to produce (E)-3-(4-acetoxy-3,5-dimethoxyphenyl)acrylic acid [24], which was further reacted with thionyl chloride under the catalyzed condition of DMF (3 drops) to provide (E)-3-(4-acetoxy-3,5-dimethoxyphenyl) acrylic chloride. Finally, the target compound I-1-I-5 was synthesized by reacting the acyl chloride 2 with the corresponding aromatic amines, respectively. In addition, the condensing reagents such as EDCI-HOBt, HATU-DIEA, or TBTU-DIEA were also taken to explore the condensation of the carboxylic acid $\mathbf{1}$ and substituted aromatic amines; however, the yields of the products were low. The target compound II-1-II-5 was obtained according to the same steps described above. Subsequently, the obtained structures were identified and characterized by ${ }^{1} \mathrm{H} \mathrm{NMR},{ }^{13} \mathrm{C} \mathrm{NMR}$, and HRMS.

3.2. Crystal Structure Analysis. The crystal structure analysis is beneficial in investigating the physical and chemical properties of the molecules. In this study, several crystal structure characteristics were also illustrated through the crystal structures and packing of molecules I-4 and II-5 (Figure 4, CCDC numbers 2095769 and 2095768). The selected bond lengths and angles are presented in Table 1, and the selected dihedral angles are shown in Table 2 . From the data, the sum of bond angles $\mathrm{C}(4)-\mathrm{C}(3)-\mathrm{H}(3), \mathrm{C}(2)-\mathrm{C}(3)-$ $\mathrm{C}(4)$, and $\mathrm{C}(2)-\mathrm{C}(3)-\mathrm{H}(3)$ in compound $\mathbf{I}-\mathbf{4}$ was $360^{\circ}$, indicating the $s p^{2}$ hybridization state of atom C(3). Similarly, the atoms $\mathrm{C}(2)$ in I-4 and $\mathrm{C}(17)$ in II-5 also adopted $s p^{2}$ hybridization state. The torsion angle of $\mathrm{C}(8)-\mathrm{C}(7)-\mathrm{O}(3)-$ 


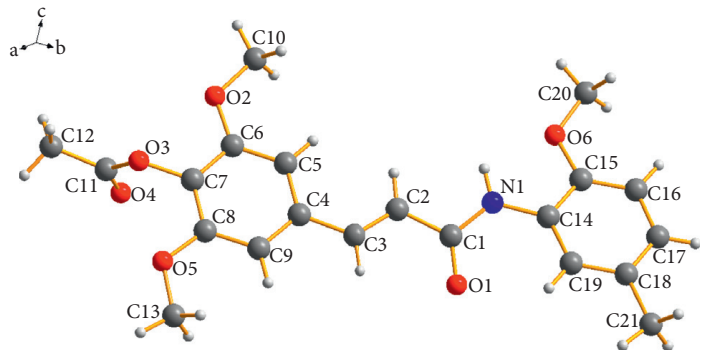

- $\mathrm{N}$

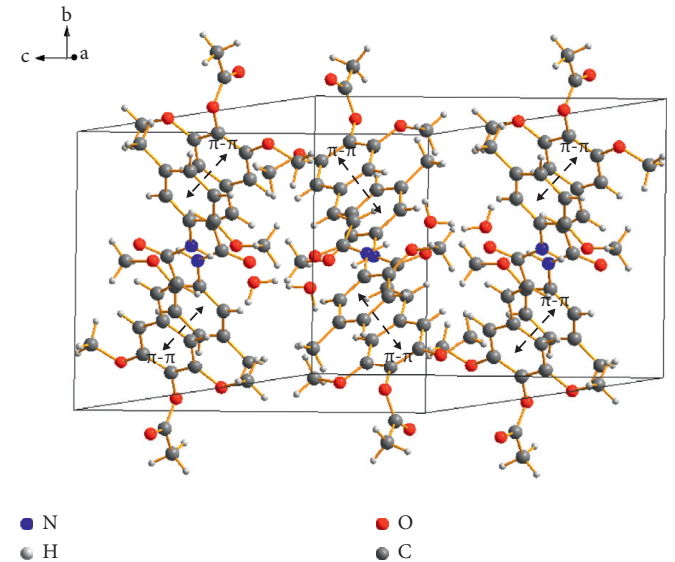

(c)

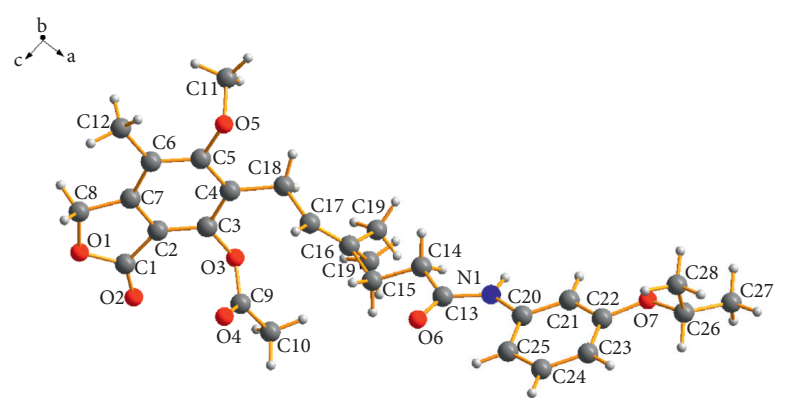

- $\mathrm{N}$

- $\mathrm{O}$

(b)

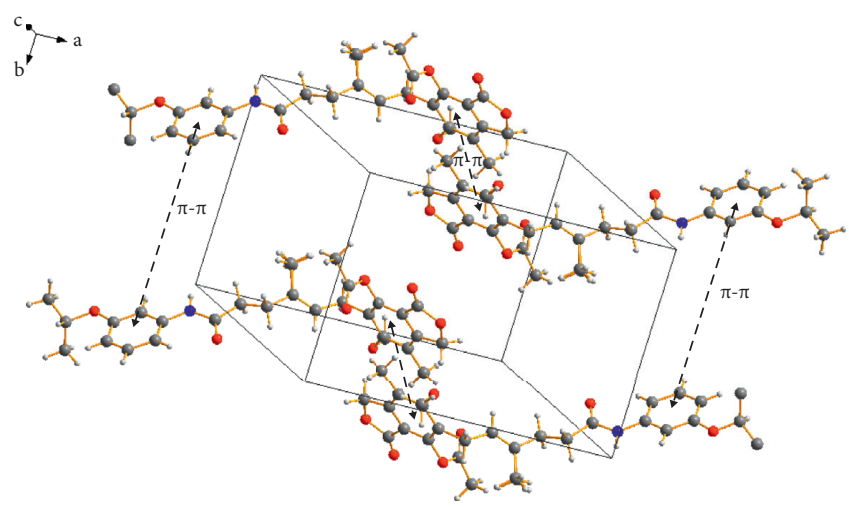

- $\mathrm{N}$

- $\mathrm{O}$

(d)

Figure 4: Crystal structures of I-4 (a) and II-5 (b), and crystal packing of I-4 (c) and II-5 (d).

TABLE 1: Selected bond lengths $(\AA)$ and bond angles $\left(^{\circ}\right)$ for the compounds I-4 and II-5.

\begin{tabular}{|c|c|c|c|}
\hline \multicolumn{2}{|c|}{ Compound I-4 } & \multicolumn{2}{|c|}{ Compound II-5 } \\
\hline Bond & Dist. $(\AA)$ & Bond & Dist. (̊) \\
\hline $\mathrm{C}(1)=\mathrm{O}(1)$ & $1.209(3)$ & $\mathrm{C}(1)=\mathrm{O}(2)$ & $1.193(4)$ \\
\hline $\mathrm{C}(1)-\mathrm{N}(1)$ & $1.332(3)$ & $\mathrm{C}(1)-\mathrm{O}(1)$ & $1.339(4)$ \\
\hline $\mathrm{C}(11)=\mathrm{O}(4)$ & $1.182(3)$ & $\mathrm{C}(3)-\mathrm{O}(3)$ & $1.385(4)$ \\
\hline $\mathrm{C}(11)-\mathrm{O}(3)$ & $1.327(3)$ & $\mathrm{C}(9)=\mathrm{O}(4)$ & $1.175(5)$ \\
\hline$C(2)=C(3)$ & $1.309(3)$ & $\mathrm{C}(9)-\mathrm{O}(3)$ & $1.354(4)$ \\
\hline $\mathrm{C}(8)-\mathrm{O}(5)$ & $1.351(3)$ & $\mathrm{C}(13)=\mathrm{O}(6)$ & $1.200(4)$ \\
\hline $\mathrm{C}(15)-\mathrm{O}(6)$ & $1.351(3)$ & $\mathrm{C}(13)-\mathrm{N}(1)$ & $1.339(4)$ \\
\hline$C(4)=C(5)$ & $1.376(3)$ & $\mathrm{C}(5)-\mathrm{O}(5)$ & $1.367(4)$ \\
\hline$C(1)-C(2)$ & $1.456(3)$ & $\mathrm{N}(1)-\mathrm{C}(20)$ & $1.390(4)$ \\
\hline$C(3)-C(4)$ & $1.444(3)$ & $C(4)=C(5)$ & $1.394(4)$ \\
\hline $\mathrm{C}(4)=\mathrm{C}(9)$ & $1.378(3)$ & $\mathrm{C}(22)-\mathrm{O}(7)$ & $1.364(3)$ \\
\hline$C(14)=C(15)$ & $1.383(3)$ & $C(16)=C(17)$ & $1.285(4)$ \\
\hline $\mathrm{C}(14)=\mathrm{C}(19)$ & $1.369(3)$ & $\mathrm{C}(17)-\mathrm{C}(18)$ & $1.482(4)$ \\
\hline$C(18)-C(21)$ & $1.484(4)$ & $C(15)-C(16)$ & $1.478(5)$ \\
\hline Bond angles & $\left(^{\circ}\right)$ & Bond angles & $\left({ }^{\circ}\right)$ \\
\hline$C(1)-C(2)-C(3)$ & $121.6(2)$ & $\mathrm{O}(1)-\mathrm{C}(1)-\mathrm{C}(2)$ & $108.4(3)$ \\
\hline$C(2)-C(3)-C(4)$ & $127.0(2)$ & $\mathrm{O}(2)-\mathrm{C}(1)-\mathrm{C}(2)$ & $130.1(3)$ \\
\hline$C(4)-C(5)-C(6)$ & $120.2(2)$ & $\mathrm{C}(1)-\mathrm{C}(2)-\mathrm{C}(7)$ & $108.1(3)$ \\
\hline$C(14)-C(15)-C(16)$ & $119.3(3)$ & $C(16)-C(17)-C(18)$ & $129.0(3)$ \\
\hline $\mathrm{O}(3)-\mathrm{C}(11)-\mathrm{O}(4)$ & $122.6(2)$ & $C(15)-C(16)-C(17)$ & $122.4(3)$ \\
\hline
\end{tabular}


TABLE 1: Continued.

\begin{tabular}{|c|c|c|c|}
\hline \multicolumn{2}{|c|}{ Compound I-4 } & \multicolumn{2}{|c|}{ Compound II-5 } \\
\hline Bond & Dist.(@) & Bond & Dist. $(\AA)$ \\
\hline $\mathrm{O}(1)-\mathrm{C}(1)-\mathrm{N}(1)$ & $122.9(2)$ & $\mathrm{O}(6)-\mathrm{C}(13)-\mathrm{N}(1)$ & $123.7(3)$ \\
\hline $\mathrm{O}(1)-\mathrm{C}(1)-\mathrm{C}(2)$ & $122.1(2)$ & $\mathrm{C}(13)-\mathrm{N}(1)-\mathrm{C}(20)$ & $129.6(3)$ \\
\hline $\mathrm{N}(1)-\mathrm{C}(1)-\mathrm{C}(2)$ & $114.9(2)$ & $C(20)-C(21)-C(22)$ & $120.9(3)$ \\
\hline $\mathrm{C}(8)-\mathrm{O}(5)-\mathrm{C}(13)$ & $117.7(2)$ & $\mathrm{C}(2)-\mathrm{C}(3)-\mathrm{C}(4)$ & $120.7(3)$ \\
\hline $\mathrm{C}(6)-\mathrm{O}(2)-\mathrm{C}(10)$ & $117.7(2)$ & $\mathrm{C}(4)-\mathrm{C}(18)-\mathrm{C}(17)$ & $114.2(3)$ \\
\hline $\mathrm{C}(15)-\mathrm{O}(6)-\mathrm{C}(20)$ & $119.2(2)$ & $\mathrm{C}(5)-\mathrm{O}(5)-\mathrm{C}(11)$ & $115.1(3)$ \\
\hline$C(3)-C(4)-C(5)$ & $121.7(2)$ & $\mathrm{C}(22)-\mathrm{O}(7)-\mathrm{C}(26)$ & $120.4(3)$ \\
\hline
\end{tabular}

TABLE 2: Selected dihedral angles $\left({ }^{\circ}\right)$ for the compounds I-4 and II-5.

\begin{tabular}{lccc}
\hline Compounds & & Groups & Dihedral angles $\left(^{\circ}\right)$ \\
\hline I-4 & Benzene ring $\{\mathrm{C}(4)-\mathrm{C}(9)\}$ & Benzene ring $\{\mathrm{C}(14)-\mathrm{C}(19)\}$ & $6.505(76)$ \\
I-4 & Benzene ring $\{\mathrm{C}(4)-\mathrm{C}(9)\}$ & Ester group $\{\mathrm{C}(11)=\mathrm{O}(4)\}$ & $88.750(188)$ \\
I-4 & Benzene ring $\{\mathrm{C}(4)-\mathrm{C}(9)\}$ & Amide group $\{\mathrm{C}(1)-\mathrm{N}(1)\}$ & $12.563(176)$ \\
I-4 & Benzene ring $\{\mathrm{C}(14)-\mathrm{C}(19)\}$ & Ester group $\{\mathrm{C}(1)=\mathrm{O}(4)\}$ & $82.625(197)$ \\
I-4 & Benzene ring $\{\mathrm{C}(14)-\mathrm{C}(19)\}$ & Amide group $\{\mathrm{C}(1)-\mathrm{N}(1)\}$ & $19.106(166)$ \\
II-5 & Benzene ring $\{\mathrm{C}(2)-\mathrm{C}(7)\}$ & Benzene ring $\{\mathrm{C}(20)-\mathrm{C}(25)\}$ & $84.911(77)$ \\
II-5 & Benzene ring $\{\mathrm{C}(2)-\mathrm{C}(7)\}$ & Ester group $\{\mathrm{C}(1)=\mathrm{O}(2)\}$ & $0.106(338)$ \\
II-5 & Benzene ring $\{\mathrm{C}(2)-\mathrm{C}(7)\}$ & Ester group $\{\mathrm{C}(9)=\mathrm{O}(4)\}$ & $74.962(214)$ \\
II-5 & Benzene ring $\{\mathrm{C}(2)-\mathrm{C}(7)\}$ & Amide group $\{\mathrm{C}(13)-\mathrm{N}(1)\}$ & $89.604(240)$ \\
II-5 & Benzene ring $\{\mathrm{C}(20)-\mathrm{C}(25)\}$ & Ester group $\{\mathrm{C}(1)=\mathrm{O}(2)\}$ & $84.918(219)$ \\
II-5 & Benzene ring $\{\mathrm{C}(20)-\mathrm{C}(25)\}$ & Ester group $\{\mathrm{C}(9)=\mathrm{O}(4)\}$ & $73.317(318)$ \\
II-5 & Benzene ring $\{\mathrm{C}(20)-\mathrm{C}(25)\}$ & Amide group $\{\mathrm{C}(13)-\mathrm{N}(1)\}$ & $12.284(211)$ \\
\hline
\end{tabular}

$\mathrm{C}(11)$ in compound $\mathbf{I}-\mathbf{4}$ was $-92.8(3)^{\circ}$, revealing that the plane of ester group was clearly vertical with the adjacent benzene ring. In compound II-5, the ester group plane consisted of $\mathrm{O}(1)-\mathrm{C}(1)-\mathrm{O}(2)$ was coplanar with the adjacent benzene ring, resulting in the existing conjugation effect between these two planes. However, the ester group plane composed of $\mathrm{O}(3)-\mathrm{C}(9)-\mathrm{O}(4)$ and the adjacent benzene ring were apparently nonplanar, with a dihedral angle of $74.830(111)^{\circ}$. The dihedral angle between the two benzene rings in compound $\mathbf{I}-4$ was $6.505(75)^{\circ}$, indicating that these two benzene rings were approximately coplanar. However, the two benzene rings in compound II-5 were nearly vertical, with a dihedral angles of $84.911(77)^{\circ}$. In addition, the amide plane was almost coplanar with the two benzene rings in compound I-4, with the dihedral angles of $13.111(183)^{\circ}$ and $19.106(159)^{\circ}$, respectively. In compound II-5, the dihedral angle between the amide plane and the benzene ring consisted of $\mathrm{C}(20)-\mathrm{C}(25)$ was $12.284(189)^{\circ}$, while the amide plane and the benzene ring consisted of $\mathrm{C}(2)-\mathrm{C}(3)$ were obviously vertical with the dihedral angle of $89.604(238)^{\circ}$. From the crystal packing, the $\pi-\pi$ interactions occurred between the benzene rings of the adjacent molecules, which strengthen the integration of the crystal molecules (Figures 4(c) and 4(d)).

3.3. Fungicidal Inhibitory Activity. The in vitro inhibitory activities of the target compounds against the common agricultural pathogens were investigated, and the results are shown in Table 3. From the data, most of the target compounds exhibited weak-to-moderate fungicidal activity against Gibberella zeae, Rhizoctonia solani, Botrytis cinerea, Cercospora circumscissa Sacc, Alternaria kikuchiana Tanaka, Colletotrichum capsici, and Alternaria sp. However, all compounds showed moderate-to-good fungicidal activity against Physalospora piricola, even better than fluopyram. For example, compounds I-1, I-4, and I-5 exhibited higher inhibitory activity than fluopyram, with the inhibitory rates of $76.2 \%, 73.3 \%$, and $73.5 \%$, respectively. It can be concluded that the compounds I-1-I-5 and II-1-II-5 displayed high selectivity for the fungicidal activity against Physalospora piricola. In terms of the relationship between the structures and the initial inhibitory activity, the structural modification had different effects on the inhibitory activities of target compounds against the different pathogens. For example, compared with the electron-withdrawing trifluoromethyl and chlorine groups, the introduction of the electron-donating methyl, methoxy, or isopropoxy group at the benzene ring was beneficial to improving the fungicidal activity of I-4 and I-5 against Physalospora piricola. For instance, the inhibition rates of compounds I-4 and I-5 were $73.3 \%$ and $73.5 \%$, respectively, which were apparently higher than those of compounds I-2 and I-3. However, this structural modification had no significant effects on the inhibitory activity of the compounds II-2-II-5 against Physalospora piricola. To further investigate the fungicidal activity of the target compounds against Physalospora piricola, the $E C_{50}$ values were measured and the results are exhibited in Table 4 . It could be found that most of the target compounds exhibited fungicidal activity equivalent to or even better than 
TABLE 3: In vitro inhibitory activity of the target compounds at $50 \mu \mathrm{g} \cdot \mathrm{mL}^{-1}$.

\begin{tabular}{|c|c|c|c|c|c|c|c|c|}
\hline \multirow{2}{*}{ Compounds } & \multicolumn{8}{|c|}{ Inhibition rate (\%) } \\
\hline & GZ & RS & $\mathrm{BC}$ & $\mathrm{CS}$ & $\mathrm{PP}$ & $\mathrm{AK}$ & $\mathrm{CC}$ & AS \\
\hline I-1 & $12.9 \pm 0.28^{* *}$ & $7.0 \pm 0.61^{* *}$ & $24.7 \pm 0.25$ & $36.5 \pm 0.59^{* *}$ & $76.2 \pm 0.66^{* *}$ & $27.9 \pm 0.34$ & $14.5 \pm 0.81^{* *}$ & $17.2 \pm 0.46^{* *}$ \\
\hline I-2 & $11.8 \pm 0.51^{* *}$ & $7.4 \pm 0.41^{* *}$ & $22.2 \pm 0.50$ & $19.7 \pm 0.51^{* *}$ & $53.9 \pm 0.83^{* *}$ & $31.0 \pm 0.43$ & $1.7 \pm 0.64^{* *}$ & $12.1 \pm 0.77$ \\
\hline I-3 & $29.9 \pm 0.32^{* *}$ & $3.2 \pm 0.30^{* *}$ & $4.2 \pm 0.55^{* *}$ & $20.1 \pm 0.81^{* *}$ & $54.8 \pm 0.79^{* *}$ & $29.1 \pm 0.67$ & $7.5 \pm 0.71^{* *}$ & $16.2 \pm 0.35^{* *}$ \\
\hline $\mathrm{I}-4$ & $41.7 \pm 0.42^{* *}$ & $2.7 \pm 0.13^{* *}$ & $22.2 \pm 0.32$ & $22.5 \pm 0.60^{* *}$ & $73.3 \pm 0.85^{* *}$ & $38.5 \pm 0.32^{* *}$ & $10.1 \pm 0.61^{* *}$ & $20.1 \pm 0.46^{* *}$ \\
\hline I-5 & $24.6 \pm 0.55^{* *}$ & $8.3 \pm 0.31^{* *}$ & $14.0 \pm 0.51^{* *}$ & $24.3 \pm 0.65^{* *}$ & $73.5 \pm 0.56^{* *}$ & $34.4 \pm 0.34^{* *}$ & $5.5 \pm 0.71^{* *}$ & $16.5 \pm 0.55^{* *}$ \\
\hline II-1 & $36.1 \pm 0.38^{* *}$ & $9.4 \pm 0.61^{* *}$ & $23.6 \pm 0.26$ & $17.3 \pm 0.51^{* *}$ & $45.2 \pm 0.11^{* *}$ & $31.1 \pm 0.34$ & $8.8 \pm 0.59^{* *}$ & $15.8 \pm 0.46^{* *}$ \\
\hline II-2 & $28.2 \pm 0.57^{* *}$ & $21.9 \pm 0.52^{* *}$ & $27.4 \pm 0.25$ & $23.0 \pm 0.60^{* *}$ & $55.9 \pm 0.39^{* *}$ & $28.5 \pm 0.24$ & $3.3 \pm 0.81^{* *}$ & $16.5 \pm 0.35^{* *}$ \\
\hline II-3 & $16.8 \pm 0.48^{* *}$ & $19.0 \pm 0.31^{* *}$ & $24.4 \pm 0.25$ & $19.7 \pm 0.51^{* *}$ & $55.3 \pm 0.59^{* *}$ & $25.6 \pm 0.83$ & $13.7 \pm 0.71^{* *}$ & $26.9 \pm 0.54^{* *}$ \\
\hline II-4 & $35.9 \pm 0.38^{* *}$ & $19.5 \pm 0.57^{* *}$ & $20.1 \pm 0.51^{* *}$ & $14.1 \pm 0.40^{* *}$ & $57.2 \pm 0.39^{* *}$ & $41.1 \pm 0.67^{* *}$ & $3.3 \pm 0.30^{* *}$ & $25.9 \pm 0.58^{* *}$ \\
\hline II-5 & $19.8 \pm 0.30^{* *}$ & $24.8 \pm 0.44^{* *}$ & $13.8 \pm 0.76^{* *}$ & $19.5 \pm 0.56^{* *}$ & $56.1 \pm 0.43^{* *}$ & $18.8 \pm 0.49^{* *}$ & $5.6 \pm 0.38^{* *}$ & $10.3 \pm 0.47^{* *}$ \\
\hline Fluopyram & 100 & $56.6 \pm 0.31^{* *}$ & $100.0^{* *}$ & $85.5 \pm 0.51^{* *}$ & $53.2 \pm 0.73^{* *}$ & $100^{* *}$ & $60.1 \pm 0.31^{* *}$ & $100^{* *}$ \\
\hline Carbendazim & 100 & 100 & $24.6 \pm 0.74$ & $27.8 \pm 0.47$ & 100 & $27.1 \pm 0.34$ & 100 & $12.4 \pm 0.49$ \\
\hline
\end{tabular}

GZ, Gibberella zeae; RS, Rhizoctonia solani; BC, Botrytis cinerea; CS, Cercospora circumscissa Sacc.; PP, Physalospora piricola; AK, Alternaria kikuchiana Tanaka; CC, Colletotrichum capsici; AS, Alternaria sp. Asterisk $\left(^{*}\right)$ means significant differences among different treatments. *, significant at the $p<0.05$ level, and ${ }^{* *}$, significant at the $p<0.01$ level compared with carbendazim.

Table 4: Medium effective concentration $\left(E C_{50}, \mu \mathrm{g} \cdot \mathrm{mL}^{-1}\right)$ values of the target compounds against Physalospora piricola.

\begin{tabular}{|c|c|c|c|}
\hline Compounds & Regression equation & Correlation coefficient $(r)$ & $E C_{50}(95 \%$ confidence interval $)$ \\
\hline I-1 & $y=1.26 x+3.35$ & 0.9918 & $20.4(17.7-23.7)^{* *}$ \\
\hline $\mathrm{I}-2$ & $y=1.00 x+3.51$ & 0.9903 & $30.7(26.2-36.0)^{* *}$ \\
\hline I-3 & $y=1.52 x+2.81$ & 0.9911 & $27.3(23.5-31.7)^{* *}$ \\
\hline I-4 & $y=1.83 x+2.33$ & 0.9908 & $28.6(24.6-33.3)^{* *}$ \\
\hline I-5 & $y=1.27 x+3.42$ & 0.9876 & $17.4(14.4-21.0)^{* *}$ \\
\hline II-1 & $y=1.78 x+1.67$ & 0.9824 & $74.2(54.1-101.7)^{* *}$ \\
\hline II-2 & $y=1.90 x+1.83$ & 0.9993 & $46.9(44.8-49.2)^{* *}$ \\
\hline II-3 & $y=1.10 x+3.44$ & 0.9835 & $25.7(20.9-31.5)^{* *}$ \\
\hline II-4 & $y=1.54 x+2.88$ & 0.9919 & $23.9(20.8-27.6)^{* *}$ \\
\hline II-5 & $y=2.46 x+1.41$ & 0.9823 & $28.8(23.2-35.7)^{* *}$ \\
\hline Fluopyram & $y=1.07 x+3.42$ & 0.9951 & $30.4(27.2-34.0)^{* *}$ \\
\hline Carbendazim & & & $<3.125$ \\
\hline
\end{tabular}

Asterisk $\left({ }^{*}\right)$ means significant differences among different treatments. ${ }^{*}$, significant at the $p<0.05$ level, and ${ }^{* *}$, significant at the $p<0.01$ level compared with carbendazim.
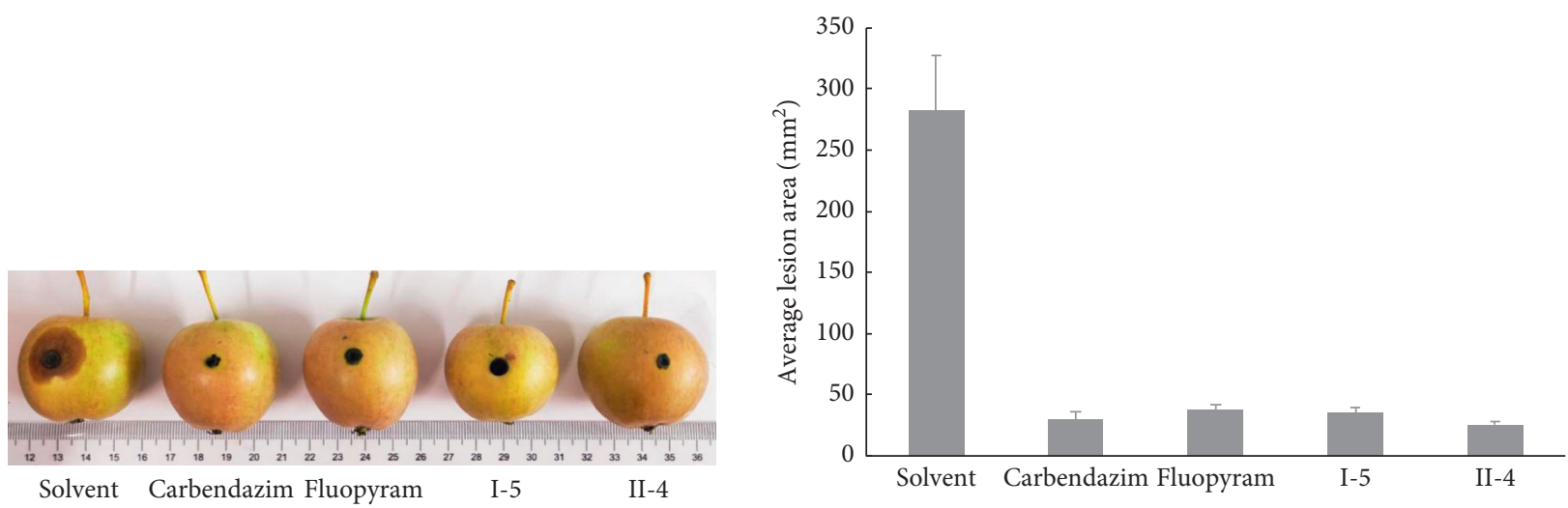

FIGURE 5: In vivo fungicidal activity of compounds I-5 and II-4 against Physalospora piricola-infected apples at $200 \mu \mathrm{g} \cdot \mathrm{mL}^{-1}$.

fluopyram, with the $E C_{50}$ values of compounds I-1, I-5, and II-4 to $20.4 \mu \mathrm{g} \cdot \mathrm{mL}^{-1}, \quad 17.4 \mu \mathrm{g} \cdot \mathrm{mL}^{-1}$, and $23.9 \mu \mathrm{g} \cdot \mathrm{mL}^{-1}$, respectively.

Subsequently, the in vivo fungicidal activity of sinapic amide I-5 and mycophenolic amide II-4 against
Physalospora piricola was performed on apples at $200 \mu \mathrm{g} \cdot \mathrm{mL}^{-1}$, and the results are displayed in Figure 5. From the data, the compounds I-5 and II-4 exhibited almost the same preventative activity as carbendazim and fluopyram at $200 \mu \mathrm{g} \cdot \mathrm{mL}^{-1}$, with the inhibition rates of carbendazim, 

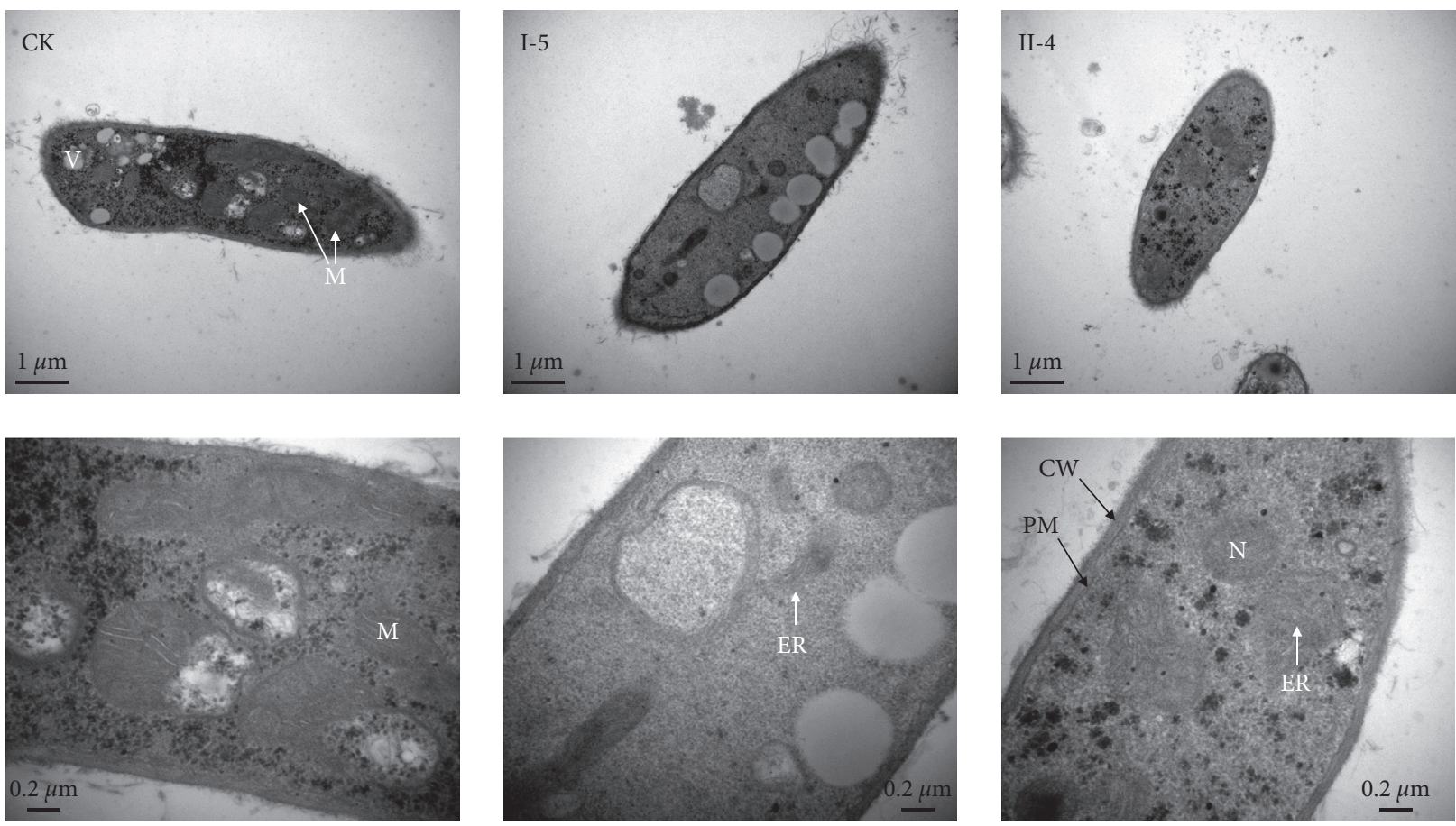

FIGURE 6: TEM investigation of Physalospora piricola hyphae treated with I-5 and II-4. CW: cell wall; PM: plasma membrane; N: nucleus; V: vacuole; M: mitochondria; ER: endoplasmic reticulum.

fluopyram, I-5, and II-4 to $90.8 \%, 86.9 \%, 87.6 \%$, and $91.1 \%$, respectively.

3.4. TEM Observation. To further explore the effects of molecules I-5 and II-4 on the hyphae, the ultrastructure of Physalospora piricola hyphae treated with distilled water, compounds I-5 and II-4 $\left(200 \mu \mathrm{g} \cdot \mathrm{mL}^{-1}\right)$, was observed on a TEM, and the results are illustrated in Figure 6. From the data, the cell wall and plasma membrane of the cell structures in the control and tested groups were normal, and mitochondria could be clearly observed in the control group. However, the mitochondria in the cell structure treated with I-5 and II-4 were blurred or even disappeared. Based on this, it could be speculated that the fungicidal activity of the target compounds against Physalospora piricola may be due to the influence of I1-I-5 and II-1-II-5 on the mitochondria in the cell structure.

\section{Conclusion}

In summary, two series of sinapic acid-derived and mycophenolic acid-derived amide derivatives were designed and synthesized. The obtained structures were characterized by ${ }^{1} \mathrm{H}$ NMR, ${ }^{13} \mathrm{C}$ NMR, HRMS, and X-ray crystal diffraction. The in vitro and in vivo fungicidal activity screening indicated that compared with other tested pathogens, most of the target compounds exhibited excellent fungicidal activity against Physalospora piricola, of which the compounds I-5 and II-4 displayed almost the same preventative activity as carbendazim and fluopyram at $200 \mu \mathrm{g} \cdot \mathrm{mL}^{-1}$. The TEM observation further revealed that the fungicidal activity of the target compounds against Physalospora piricola may be due to the influence on the mitochondria in the cell structure.

\section{Data Availability}

The data used to support the findings of this study are included within the article and the supplementary information file(s). Crystallographic data for the structures reported in this manuscript have been deposited with the Cambridge Crystallographic Data Centre under the CCDC numbers: 2095769 (compound I-4) and 2095768 (compound II-5). Copies of these data can be obtained free of charge from http://www.ccdc.cam.ac.uk/ data_request/cif.

\section{Conflicts of Interest}

There are no conflicts of interest to declare.

\section{Acknowledgments}

This research was supported by the National Natural Science Foundation of China (No. 32001929), Shandong Provincial Agricultural Science and Technology Project (Park Indus-try Upgrade Project) (No. 2019YQ037), the National Innovation and Entrepreneurship Training Program for College Students (No. 202110447013, 202110447032), and the Innovation and Entrepreneurship Training Program for College Students of Liaocheng University (No. CXCY2020Y116).

\section{Supplementary Materials}

The supporting information contained X-ray crystal data of the compounds I-4 and II-5, and ${ }^{1} \mathrm{H}$ NMR, ${ }^{13} \mathrm{C}$ NMR, and 
HRMS spectra of target compounds I-1-I-5 and II-1-II-5. (Supplementary Materials)

\section{References}

[1] C. Yan, J. Dong, Y. Liu, Y. Li, and Q. Wang, "Target-directed design, synthesis, antiviral activity, and SARs of 9-substituted phenanthroindolizidine alkaloid derivatives," Journal of Agricultural and Food Chemistry, vol. 69, no. 27, pp. 7565-7571, 2021.

[2] M. Kabbage, J. S. Piotrowski, E. Thill et al., "Poacic acid suppresses dollar spot and snow mould in amenity turfgrass," Plant Pathology, vol. 69, no. 1, pp. 112-119, 2020.

[3] J.-K. Zhu, J.-M. Gao, C.-J. Yang et al., "Design, synthesis, and antifungal evaluation of neocryptolepine derivatives against phytopathogenic fungi," Journal of Agricultural and Food Chemistry, vol. 68, no. 8, pp. 2306-2315, 2020.

[4] J. G. Zheng, T. T. Liu, Z. X. Guo et al., "Fumigation and contact activities of 18 plant essential oils on Villosiclava virens, the pathogenic fungus of rice false smut," Scientific Reports, vol. 9, pp. 1-10, 2019.

[5] S. X. Guo, F. He, B. A. Song, and J. Wu, "Future direction of agrochemical development for plant disease in China," Food and Energy Security, vol. 10, pp. e293-1-e293-16, 2021.

[6] X. Hua, W. Liu, Y. Chen et al., "Synthesis, fungicidal activity, and mechanism of action of pyrazole amide and ester derivatives based on natural products l-serine and waltherione alkaloids," Journal of Agricultural and Food Chemistry, vol. 69, no. 38, pp. 11470-11484, 2021.

[7] J. W.-H. Li and J. C. Vederas, "Drug discovery and natural products: end of an era or an endless frontier?" Science, vol. 325 , no. 5937, pp. 161-165, 2009.

[8] T. Rodrigues, D. Reker, P. Schneider, and G. Schneider, "Counting on natural products for drug design," Nature Chemistry, vol. 8, no. 6, pp. 531-541, 2016.

[9] L. W. K. Moodie, K. Sepčić, T. Turk, R. Frangež, and J. Svenson, "Natural cholinesterase inhibitors from marine organisms," Natural Product Reports, vol. 36, no. 8, pp. 1053-1092, 2019.

[10] D. J. Newman and G. M. Cragg, "Natural products as sources of new drugs over the nearly four decades from 01/1981 to 09/ 2019," Journal of Natural Products, vol. 83, no. 3, pp. 770-803, 2020.

[11] J. Liu, S. Lu, J. Feng et al., "Enantioselective synthesis and antifungal activity of C18 polyacetylenes," Journal of Agricultural and Food Chemistry, vol. 68, no. 7, pp. 2116-2123, 2020.

[12] C. L. Liu, A. Y. Guan, Z. N. Li et al., "Preparation and application of benzopyrone compounds with insecticidal and fungicidal activity," vol. 6, 2007 in Chinese, Article ID 200310105079.

[13] S. Anand and P. Srivastava, "Optimization strategies for purification of mycophenolic acid produced by Penicillium brevicompactum," Applied Biochemistry and Biotechnology, vol. 191, no. 2, pp. 867-880, 2020.

[14] R. Freedman, R. Yu, A. W. Sarkis, and L. Hedstrom, "A structural determinant of mycophenolic acid resistance in eukaryotic inosine $5^{\prime}$-monophosphate dehydrogenases," Protein Science, vol. 29, no. 3, pp. 686-694, 2020.

[15] P. Chen, C. Liu, J. Hu, H. Zhang, and R. Sun, "Design, synthesis and fungicidal activity studies of 3-ferrocenyl-Nacryloylmorpholine," Journal of Organometallic Chemistry, vol. 854, pp. 113-121, 2018.
[16] W. C. Liu and C. L. Liu, "Novel fungicide flumorph (SYPL190) with high activity," Pesticides, vol. 41, pp. 8-11, 2002, in Chinese.

[17] C. W. Mu, H. Z. Yuan, N. Li et al., "Synthesis and fungicidal activities of a novel series of 4-[3-(pyrid-4-y1)-3-substituted phenyl acryloyl]morpholine," Chemical Journal of Chinese Universities, vol. 28, pp. 1902-1906, 2007.

[18] Y. Xiao, X. Yang, B. Li et al., "Design, synthesis and antifungal/insecticidal evaluation of novel cinnamide derivatives," Molecules, vol. 16, no. 11, pp. 8945-8957, 2011.

[19] S. E. Lee, N. E. Mahoney, and B. C. Campbell, "Inhibition of aflatoxin B1 biosynthesis by piperlongumine isolated from Piper longum L." Journal of Microbiology and Biotechnology, vol. 12, pp. 679-682, 2002.

[20] Y. S. Moon, W. S. Choi, E. S. Park et al., "Antifungal and antiaflatoxigenic methylenedioxy-containing compounds and piperine-like synthetic compounds," Toxins, vol. 8, pp. 2401-240-10, 2016.

[21] X. Hua, N. Liu, S. Zhou et al., "Design, synthesis, and biological activity of novel aromatic amide derivatives containing sulfide and sulfone substructures," Engineering, vol. 6, no. 5, pp. 553-559, 2020.

[22] W. R. Liu, X. W. Hua, S. Zhou et al., "Design, synthesis and biological activity of $\mathrm{N}$-sulfonyl aromatic amide derivatives," Chinese Journal of Structural Chemistry, vol. 40, pp. 666-674, 2021.

[23] X. Hua, W. Liu, Y. Su et al., "Studies on the novel pyridine sulfide containing SDH based heterocyclic amide fungicide," Pest Management Science, vol. 76, no. 7, pp. 2368-2378, 2020.

[24] F. Allais, P.-H. Ducrot, and S. Martinet, "Straightforward total synthesis of 2-O-Feruloyl-1-malate, 2-O-Sinapoyl-1-malate and 2-O-5-Hydroxyferuloyl-1-malate," Synthesis, vol. 2009, no. 21 , pp. $3571-3578,2009$. 